\title{
EZB-Politik mit restriktivem Unterton
}

Keine der beiden Aufschwungsphasen, die die Europäische Zentralbank (EZB) begleitet hat, konnte sich voll entfalten; sie kamen vorher zu einem abrupten Ende. Ursächlich waren in beiden Fällen primär exogene Schocks. Vergleichbare Schocks hätten im Vorfeld der Währungsunion mit hoher Wahrscheinlichkeit zu Währungsturbulenzen geführt - mit einer deutlichen Aufwertung der D-Mark sowie Zinserhöhungen in den abwertenden Ländern, wodurch der restriktive Impuls der negativen Schocks noch verstärkt worden wäre. Dass derartige Währungsturbulenzen ausbleiben, ist für sich genommen ein wichtiger positiver Aspekt des Euroraums. Es stellt sich jedoch darüber hinaus die Frage, ob die EZB auf die Folgen der exogenen Schocks angemessen reagiert hat.

\section{Einleitung}

Kurz nachdem die EZB im Januar 1999 die geldpolitischen Zügel für die damals elf Länder des Euroraums übernahm, war sie in kurzer Abfolge mit mehreren Schocks konfrontiert: den Nachwirkungen der Asienkrise 1997/1998, der Verdreifachung des Ölpreises 1999, dem synchronen weltwirtschaftlichen Abschwung 2001 mit Rezessionen in den Vereinigten Staaten und Japan, den Terroranschlägen vom 11. September 2001 und den damit einhergehenden sowie durch den drohenden Irakkrieg verstärkten geopolitischen Unsicherheiten. In den Jahren 2001 bis 2003 nahm das Bruttoinlandsprodukt (BIP) im Euroraum mit einer jährlichen Durchschnittsrate von nur $1,2 \% \mathrm{zu}$, und das obwohl die EZB den Leitzins bis Juni 2003 um 2,75 Prozentpunkte auf $2 \%$ senkte; die Arbeitslosenquote stieg um einen Prozentpunkt auf knapp $9 \%$. Der zweite wirtschaftliche Einbruch in diesem Jahrzehnt - die aktuelle Finanzmarkt- und Weltwirtschaftskrise lässt die wirtschaftliche Schwächephase zu Beginn des Jahrzehnts als harmlose geldpolitische Übung erscheinen. Mitte 2009 liegt der geldpolitische Leitzins bei $1 \%$ und die EZB hat mittlerweile Maßnahmen der quantitativen Lockerung ergriffen. Das BIP dürfte 2009 und 2010 schrumpfen mit Raten von 4,5\% bzw. 0,6\% - und die Arbeitslosenquote wird vermutlich bis Ende 2010 auf über $12 \%$ klettern.

Hat die EZB auf die beiden wirtschaftlichen Einbrüche angemessen reagiert? Ausgehend von dieser Frage werden im Folgenden zunächst die geldpolitische Strategie der EZB diskutiert sowie deren Veränderung im Laufe der Zeit (Abschnitt 2). Es folgt ein Überblick über die beiden Wirt- schaftseinbrüche 2001/2002 sowie 2009/ 2010 und die Rolle, die die europäische Geldpolitik dabei spielte (Abschnitt 3). Den Einfluss der Geldpolitik auf die mittelfristige Entwicklung erörtert Abschnitt 4. Abschnitt 5 zieht ein Fazit.

\section{2 \\ Wandel der geldpolitischen Strategie und Kommuni- kation}

Die im Oktober 1998 von der EZB festgelegte geldpolitische Strategie und ihre Kommunikation waren für die Länder des Euroraums in vielerlei Hinsicht innovativ und vielversprechend. So gab sich die EZB ein explizites Inflationsziel und reihte sich damit de facto in die Gruppe von führenden Zentralbanken ein, die mit dem Inflation Targeting eine Strategie verfolgen, die gegenwärtig gemeinhin als die Beste angesehen wird (Bernanke 2004; Svensson 2008). Die geldpolitische Strategie des Inflation Targeting ist dadurch gekennzeichnet, dass ein offizielles Inflationsziel im Voraus angekündigt wird und sich die Zentralbank explizit dem primären Ziel der Preisstabilität verpflichtet (Bernanke/Mishkin 1997; Svensson 1997). Weitere wichtige Bestandteile dieser Strategie bestehen in der ausführlichen öffentlichen Darlegung der Entscheidungsgrundlage und der Beweggründe für die aktuelle Geldpolitik. Eine hohe Rechenschaftspflicht ergibt sich dabei aus der expliziten Vorgabe eines quantifizierten Inflationsziels. Die EZB hob frühzeitig hervor, dass sie die Inflationsaussichten sowohl anhand monetärer Indikatoren, wie der Geldmenge, der Kreditentwicklung und der Aktienkurse, als auch anhand realwirtschaftlicher Indikatoren, wie der Produktionslücke und der
Arbeitsmarktentwicklung, abschätzen würde. Ein hohes Maß an Transparenz deutete sich auch durch die monatlichen Pressekonferenzen an, in denen sich der EZBPräsident den Journalisten stellen würde. Einige wichtige Aspekte der Strategie mussten allerdings im Laufe der Zeit nachgebessert werden. Dies betraf insbesondere die nebulöse Quantifizierung des Inflationsziels und die verbale Hervorhebung der Geldmenge als erste Säule der geldpolitischen Strategie.

\subsection{NEBULÖSES INFLATIONSZIEL}

Die ursprüngliche EZB-Definition von Preisstabilität als „Anstieg des Harmonisierten Verbraucherpreisindex (HVPI) für das Euro-Währungsgebiet von unter $2 \%$ gegenüber dem Vorjahr" lieferte einen erheblichen Interpretationsspielraum. In einer Rede im Oktober 1999 stellte der erste EZB-Präsident Duisenberg klar, dass das Wort Anstieg in der Definition signalisieren sollte, dass auch ein Rückgang des Preisniveaus nicht mit Preisstabilität zu vereinbaren sei. So erklärte er: „The use of the word, increases' in the definition imposes a floor of at least zero for the lower bound" (Duisenberg 1999). Und auch Vizepräsident Noyer (2000) betonte: „The phrase ,below $2 \%$ ' clearly delineates the upper bound for the rate of measured inflation in the HICP, which is consistent with price stability. At the same time, the use of the word ,increase' in the definition clearly

Silke Tober, Dr., ist Wissenschaftlerin im
Institut für Makroökonomie und Konjunktur-
forschung (IMK) in der Hans-Böckler-Stiftung.
Arbeitsschwerpunkte: Makroökonomische
und insbesondere geldpolitische Analysen.
e-mail: Silke-Tober@boeckler.de


signals that deflation, i. e. prolonged declines in the level of the HICP, would not be deemed consistent with price stability".

Zahlreiche Beobachter schlossen aus diesen Ausführungen, dass der EZB ein Zielkorridor zwischen $0 \%$ und $2 \%$ vorschwebe. Diese Einschätzung stand allerdings in Widerspruch zu dem impliziten Inflationsziel, das aus den Vorgaben für die Berechnung des Referenzwerts für das Geldmengenwachstum abgeleitet werden konnte; dieses Inflationsziel lag bei 1,5\%. Bei Symmetrie ergab sich eine Untergrenze von $1 \%$. Eine über Null liegende Untergrenze ließ sich auch deshalb vermuten, weil Vertreter der EZB mehrfach auf die aus verschiedenen Studien bekannten Messprobleme der Inflation hinwiesen, die infolge einer unzureichenden Erfassung von Qualitätsverbesserungen eine Verzerrung nach oben erzeugen.

Infolge der Mehrdeutigkeit und Asymmetrie war die Zieldefinition der EZB als Richtschnur in Lohnverhandlungen nur begrenzt einsetzbar. Aber gerade in diesem Bereich ist eine klare Vorgabe erforderlich, soll ein Konflikt zwischen Geld- und Lohnpolitik vermieden werden. Ein solcher Konflikt ist vorprogrammiert, wenn sich die Lohnentwicklung am mittelfristigen Produktivitätsfortschritt zuzüglich der einzigen quantitativen Vorgabe der EZB - der Obergrenze von $2 \%$ - orientiert, die EZB aber letztlich ein mittelfristiges Inflationsziel von 1,5\% verfolgt und andere Marktteilnehmer gar ein Inflationsziel von $1 \%$ vermuten.

Bei der Präzisierung ihres Inflationsziels im Frühjahr 2003 nahm die EZB ihre ursprüngliche Definition als Ausgangspunkt. So strebt sie in der mittleren Frist weiterhin einen „Anstieg des Harmonisierten Verbraucherpreisindex (HVPI) für das Euro-Währungsgebiet von unter $2 \%$ gegenüber dem Vorjahr" an. Das Problem der bisher nicht eindeutigen Untergrenze des Inflationsziels wurde dadurch gelöst, dass als Ziel ausdrücklich festgelegt wurde, mittelfristig eine Preissteigerungsrate von nahe $2 \%$ beizubehalten. Das Ziel einer Inflationsrate unter, aber nahe $2 \%$ wird mittlerweile allgemein als ein Inflationsziel von 1,9\% interpretiert (Gemeinschaftsdiagnose 2008 , S. 81) und liefert damit eine konkrete Orientierungsgröße.

Vielfach wurde in den ersten Jahren kritisiert, dass die EZB ihre internen Inflationsprognosen nicht veröffentlichte. Im Dezember 2000 präsentierte die EZB dann erstmals eine bedingte BIP- und Inflationsprognose des Stabes des Eurosystems. ${ }^{1}$ Sie wird seither zwei Mal im Jahr veröffentlicht (Dezember und Juni) und ergänzt um halbjährliche Prognosen des EZB-Stabs (März und September). Dies war ein wichtiger Schritt zur Nachvollziehbarkeit der geldpolitischen Entscheidungen und rundete die geldpolitische Strategie des Inflation Targeting ab.

\subsection{STELLENWERT DER GELDMENGE}

Bei der Neuformulierung ihrer geldpolitischen Strategie im Frühjahr 2003 beseitigte die EZB zudem die irreführende Darstellung der Bedeutung der Geldmenge in der geldpolitischen Strategie und trug mit der Betonung des mittelfristigen Charakters der Geldmenge ihrer faktischen Geldpolitik Rechnung. Der Geldmenge und den anderen monetären Aggregaten kommt seither die Rolle zu, die sie auch erfüllen können, nämlich die als begleitende Informationsvariablen für die mittelfristigen Preisperspektiven.

Ursprünglich wurde die herausgehobene Stellung der Geldmenge betont, was im Widerspruch dazu stand, dass die EZB trotz einer Überschreitung des Referenzwertes für die Entwicklung der Geldmenge M3 die Zinsen in den Jahren 1999, 2001 und 2002 nicht etwa erhöhte, sondern senkte. Die EZB trug so zu einer gewissen Verwirrung bei, indem sie die Bedeutung der Geldmenge als erste Säule der geldpolitischen Strategie herausstrich, anstatt ihre Komplementärfunktion für die mittlere Frist zu betonen. In der mittleren Frist kann die Geldmengenentwicklung durchaus wichtige Informationen liefern, sofern ein stabiler Zusammenhang zwischen Geldmenge, Preisen und Bruttoinlandsprodukt besteht. Kurzfristig - über einige Jahre hinweg - hat die Geldmenge allerdings infolge möglicher Portfolioumschichtungen keine guten Indikatoreigenschaften (Schumacher/Tober 1999a; Svensson/Gerlach 2002).

Ökonometrische Studien weisen tatsächlich darauf hin, dass die Geldnachfrage im Euroraum langfristig stabil sein dürfte (Brand/Cassola 2000; Coenen/Vega 1999; Schumacher/Tober 1999a). In der kurzen Frist treten allerdings Abweichungen der Geldnachfrage von dem langfristig stabilen Pfad auf, sodass sich die Zentralbank schon aus diesem Grund kurzfristig nicht primär an der Geldmenge orientieren kann. Zwei- tens spricht gegen eine starke Orientierung an der Geldmenge, dass sie bestenfalls ein adäquater Frühindikator dafür sein kann, ob von der Geldpolitik eine destabilisierende Wirkung auf den Geldwert ausgeht. Störungen, die ihren Ursprung in der realen Sphäre haben, zeigt die Geldmenge erst an, wenn sie bereits realwirtschaftliche Wirkungen entfaltet haben - und damit zu spät für einen frühzeitigen stabilisierenden Eingriff der Geldpolitik.

Trotz der theoretischen und empirischen Einwände gegen eine kurzfristige Orientierung an der Geldmenge gab es im Vorfeld der Europäischen Währungsunion Stimmen, die eine prominentere Rolle der Geldmenge in der geldpolitischen Strategie der EZB bzw. gar eine reine Geldmengensteuerung forderten (SVR 1998, S. 6f.; Neumann 1998). Damit sollte auch an die (verbale) Tradition der Deutschen Bundesbank angeknüpft werden, die jedes Jahr einen bestimmten Anstieg der Geldmenge als Zwischenziel festlegte. Allerdings hatte die Bundesbank ihr Zwischenziel in der Hälfte aller Jahre verfehlt, weshalb beispielsweise Bernanke/Mihov (1997) sie als ,impliziten“ Inflation Targeter bezeichnen.

Problematisch war nicht nur die ursprüngliche Hervorhebung der Geldmenge als „erste Säule“ der geldpolitischen Strategie, sondern auch die festgelegte Höhe des Referenzwerts für die Entwicklung der Geldmenge M3. Der Referenzwert war mit $4,5 \%$ von Anfang an eher niedrig angesetzt. Die 4,5\% setzten sich zusammen aus 2,25\% für den trendmäßigen Zuwachs des Bruttoinlandsproduktes (BIP), 1,5\% für den Anstieg des Preisniveaus und 0,75\% für die trendmäßige Abnahme der Umlaufgeschwindigkeit. ${ }^{2}$ Der relativ schwache Rückgang der Umlaufsgeschwindigkeit von M3, den die EZB berücksichtigte, kam durch die Wahl des zugrunde gelegten Zeitraums (1980 bis 1999 und 1992 bis 1999) zustande. Demgegenüber ergibt sich für die Zeiträume 1991 bis 2000 sowie 1995

\footnotetext{
1 Das Eurosystem besteht aus den nationalen Zentralbanken und der EZB, wobei im EZB-Rat sämtliche Präsidenten der nationalen Zentralbanken vertreten sind.

2 Die EZB gibt für die einzelnen Raten Spannen an, aus denen die konkreten Werte hier als Mittelwert berechnet sind. Im Einzelnen sind dies höchstens $2 \%$ bei dem Anstieg des Preisniveaus, $2 \%-2,5 \%$ bei dem trendmäßigen Anstieg des Bruttoinlandsproduktes und $0,5 \%-1 \%$ bei dem Zuschlag für die trendmäßige Abnahme der Umlaufgeschwindigkeit (Europäische Zentralbank 1999, S. 10f.).
} 
bis 2000 eine trendmäßige Abnahme der Umlaufgeschwindigkeit um jahresdurchschnittlich etwa 1,3\%; für den längeren Zeitraum 1980 bis 1999 liegt sie bei rund $1 \%$ (Gemeinschaftsdiagnose 1999, S. 38ff.; Tober 2000, S. 803f.). Auch das Trendwachstum war niedrig angesetzt. Schätzungen des Potenzialwachstums lagen Anfang des Jahrzehnts eher bei $2,5 \%$ als bei $2,25 \%$. So ging der International Monetary Fund (IMF) im Jahre 2000 von einem Trend- bzw. Potenzialwachstum im Euroraum in Höhe von 2,5 \% aus (IMF 2000a, S. 8), mehrere andere ökonometrische Verfahren deuteten auf einen Anstieg von $2,4 \%$ hin (Schumacher/Tober 1999b, S. 16f.), und selbst die EZB konstatierte, dass die Unsicherheiten der Schätzungen des Potenzialwachstums eher auf der oberen Seite lagen. ${ }^{3}$ Das Problem eines relativ niedrigen Referenzwerts für das Geldmengenwachstum besteht weiterhin, zumal er angesichts der Präzisierung des Inflationsziels im Jahre 2003 selbst bei der unrealistisch niedrigen Annahme eines trendmäßigen Rückgangs der Umlaufgeschwindigkeit um 0,75 \% lediglich Raum für einen trendmäßigen Anstieg des Bruttoinlandsproduktes im Euroraum in Höhe von knapp $1,9 \%$ bietet.

\subsection{FAZIT: STRATEGIE KONZEP- TIONELL GELUNGEN, ABER MIT RESTRIKTIVEM BIAS IN DER PRAXIS}

Mit ihren Pressekonferenzen und Auftritten vor dem Europäischen Parlament sowie der Veröffentlichung ihrer Inflations- und Wachstumsprognose zeigt sich die EZB in ihrer Kommunikation deutlich zugänglicher als es die Bundesbank gewesen war, auch wenn ihre Rechenschaftspflicht gegenüber dem Parlament weniger ausgeprägt ist als die der US-amerikanischen Zentralbank (Fed). Ihre geldpolitische Strategie zeigt alle Merkmale einer Zentralbank, die Inflation Targeting betreibt. Dass die EZB sich nach wie vor gegen diese Bezeichnung sträubt, lässt sich nur mit Befindlichkeiten einzelner Mitglieder erklären. Regelgebundene Strategien können sich positiv auf die Transparenz, Rechenschaftspflicht und Glaubwürdigkeit der Geldpolitik auswirken. Für die Transparenz und Glaubwürdigkeit der Geldpolitik ist es dabei entscheidend, dass die Zentralbank "tut, was sie sagt, und sagt, was sie tut" (Artis et al. 1998, S. 1824). ${ }^{4}$ Auch aus diesem Grund war es wichtig, dass die EZB den
Stellenwert der Geldmenge im Jahre 2003 relativierte.

Die geldpolitische Strategie wies von Beginn an einen gewissen restriktiven Bias der EZB auf. Dieser zeigte sich erstens daran, dass Preisstabilität zunächst mit ausschließlichem Bezug auf eine Obergrenze definiert wurde. Zweitens wurde der Referenzwert für den Anstieg der Geldmenge relativ niedrig angesetzt. Und drittens stellte die EZB mehrfach klar, dass sie mit der Wahrung der Preisstabilität ihren Auftrag als erfüllt ansieht; eine darüber hinausgehende Unterstützung der Ziele der Gemeinschaft, wie es der EU-Vertrag vorsieht, sofern das primäre Ziel der Geldwertstabilität nicht gefährdet ist, sieht sie aber nicht als ihre Aufgabe an. Dieser restriktive Bias hatte im ersten Jahr des Euroraums keine praktische Konsequenz. Um die EZB aus der Schusslinie zu nehmen, hatten die nationalen Zentralbanken als Reaktion auf die sich drohende Abschwächung infolge der Asienkrise bereits im Dezember 1998 die Zinsen auf 3 \% gesenkt; die EZB legte im April 1999 mit einer Senkung um 0,5 Prozentpunkte nach. Trotz abgeschwächter Exporte nahm das Bruttoinlandsprodukt 1999 um 2,4\% zu. ${ }^{5}$ In den Folgejahren zeigte sich dieser Bias dann allerdings und trug mit dazu bei, dass der Euroraum von 2001 bis 2004 eine mehrjährige Schwächephase erlebte. Ähnlich zögerlich reagierte die EZB dann auch auf die aktuelle Weltwirtschaftskrise.

\section{3 \\ Das jähe Ende der zwei Aufschwungsphasen}

In den Jahren 2001 und 2008 brach jeweils ein Aufschwung ab, der sich noch nicht voll entfaltet hatte bzw. der noch nicht an seine inneren Grenzen gestoßen war. Den jeweiligen Abschwungsphasen war gemein, dass sie erstens von exogenen Schocks eingeleitet wurden, dass zweitens die EZB die Zinsen nochmals erhöhte, als sich Schwächetendenzen der Weltwirtschaft und des Euroraums bereits deutlich abzeichneten, und dass die EZB drittens auf die wirtschaftliche Schwäche nur langsam reagierte.

\subsection{ABSCHWUNG 2001/2002}

Für den Abbruch des Aufschwungs im Euroraum 2000/2001 waren in erster Linie vier Faktoren verantwortlich: Die drasti- sche Verteuerung von Rohöl, der Einbruch der Gewinnerwartungen im Informationstechnologie (IT)-Sektor, die Rezession in den USA und die geldpolitische Straffung der EZB. Der Ölpreis schnellte 1999/2000 empor; in Euro gerechnet übertraf er im Durchschnitt des Jahres 2000 den Wert von 1998 um 170 \%, den Wert von Januar 1999 sogar um $220 \%$. Im Laufe des Jahres 2000 nahm auch der Internetboom ein Ende mit sinkenden Aktienkursen und einem Einbruch der Gewinnerwartungen im IT-Sektor. Diese zwei Faktoren bewirkten ihrerseits, dass es Ende 2000 in den USA nach zehn Aufschwungjahren nicht nur zu einer wirtschaftspolitisch gewünschten Abschwächung des Wachstumstempos kam, sondern im Jahre 2001 zu einer Rezession; die Arbeitslosigkeit stieg von knapp $4 \%$ Mitte 2000 bis Ende 2001 auf 5,8 \%. Die EZB ihrerseits hatte den geldpolitischen Leitzins im Laufe des Jahres 2000 um 1,75 Prozentpunkte auf $4,75 \%$ angehoben (Abbildung 1). Im Euroraum kam es zu einer deutlichen Erwartungseintrübung; die Investitionen brachen Ende 2000 ein, ab Anfang 2001 stagnierten die Exporte, und der Konsum war trotz Steuererleichterungen in zahlreichen Ländern schwach.

Für sich genommen hätte die geldpolitische Restriktion den deutlichen Abschwung im Euroraum nicht bewirkt, zumal der Euro im Jahr 2000 stark abwertete und die monetären Rahmenbedingungen aufhellte. Zudem erwartete die EZB noch im Dezember 2000 für 2001 und 2002 einen Anstieg des BIP im Euroraum um 3,1 \% bzw. $3 \%$ (Europäische Zentralbank 2000, S. 54ff.). Da sie mit dieser Fehlprognose nicht allein stand, ${ }^{6}$ kann man ihr kei-

3 "The uncertainties surrounding the estimates of medium-term development of potential output growth in the euro area have become skewed to the upside" (Europäische Zentralbank 2000).

4 Da auch die Bundesbank Verfehlungen des Geldmengenziels hinnahm, um ihr Inflationsziel zu erreichen, kommen beispielsweise Bernanke/Mihov (1997) zu dem Schluss, dass selbst die Bundesbank eher als „inflation-targeter " statt als Geldmengensteuerin zu bezeichnen ist.

5 Dass die Exporte immerhin noch mit einer Rate von 4,5\% zunahmen, ist auch darauf zurückzuführen, dass die US-amerikanische Wirtschaftspolitik stark expansiv wirkte.

6 Die OECD erwartete im Dezember 2000 einen Anstieg des BIP um 3,1 \% für 2001 und 2,8 \% für 2002 (OECD 2000), der IMF hatte im Oktober 2000 sogar noch einen Anstieg um 3,4\% für 2001 vorhergesagt (IMF 2000b), die Wirtschaftsforschungsinstitute einen von 2,8\% (Gemeinschaftsdiagnose 2000) 
nen Vorwurf daraus machen, dass sie nicht bereits Ende 2000 die geldpolitischen Zügel lockerte. Gerechtfertigt scheint demgegenüber die Kritik, dass die EZB die Zinsen im Jahre $2000 \mathrm{zu}$ stark angehoben hatte. Nachdem die EZB bereits im November 1999 die im April 1999 im Zuge der Asienkrise vollzogene Zinssenkung zurückgenommen hatte, erhöhte sie den geldpolitischen Leitzins 2000 um insgesamt 1,75 Prozentpunkte auf 4,75\% und ging damit zu einem restriktiven Kurs über. Die letzte Zinserhöhung erfolgte im Oktober 2000, als die europäische Wirtschaft sich bereits deutlich abgeschwächt hatte und die wirtschaftliche Entwicklung in dem größten Mitgliedstaat, Deutschland, gar zum Stillstand gekommen war. Dies dürfte umso gravierender gewesen sein, als zumindest ökonometrischen Untersuchungen für Deutschland zufolge geldpolitische Maßnahmen im Abschwung stärker wirken als im Aufschwung (Kuzin/Tober 2004; Kakes 2000).

In dieser deutlichen geldpolitischen Straffung trotz sich abzeichnender weltwirtschaftlicher Abschwächung kam die restriktive Grundhaltung der EZB zum Ausdruck: Da die EZB mittelfristig nur ein geringes Wachstum von 2,25 \% als kompatibel mit Preisniveaustabilität erachtete, wollte sie das Wachstumstempo deutlich dämpfen, um es in Einklang mit dem Trendwachstum der Vergangenheit zu bringen. Auch reagierte die EZB auf die drastische Erhöhung der Importpreise, die sich in einem spürbaren Preisniveauschub bei den Verbraucherpreisen niederschlug. Dieser Preisschub war aber maßgeblich auf den Rohölpreis zurückzuführen und wurde durch die Folgen zweier Tierseuchen, die deutliche Abwertung des Euro und die Erhöhung staatlich beeinflusster Preise verstärkt. Da die Lohnentwicklung moderat war und es keine nennenswerten Anzeichen für eine Verstärkung des Lohndrucks durch Engpässe auf dem Arbeitsmarkt oder Nachschlagsforderungen der Arbeitnehmer als Reaktion auf den Schub bei den Verbraucherpreisen gab, hätten weder das kräftige Wachstum in Höhe von 2,9 \% bzw. 3,9\% in den Jahren 1999 und 2000 noch der Ölpreisschock einen restriktiven Kurs der Geldpolitik erfordert: Ein länger währender kräftiger Aufschwung hätte bei moderater Lohnentwicklung zu einer Erhöhung der Beschäftigung und einem Abbau der Arbeitslosigkeit führen können, während der Preisniveauschub als Einmaleffekt

Abb. 1: Leitzinsen im Euroraum und in den USA 1991 bis $2009^{1}$ - in \% -

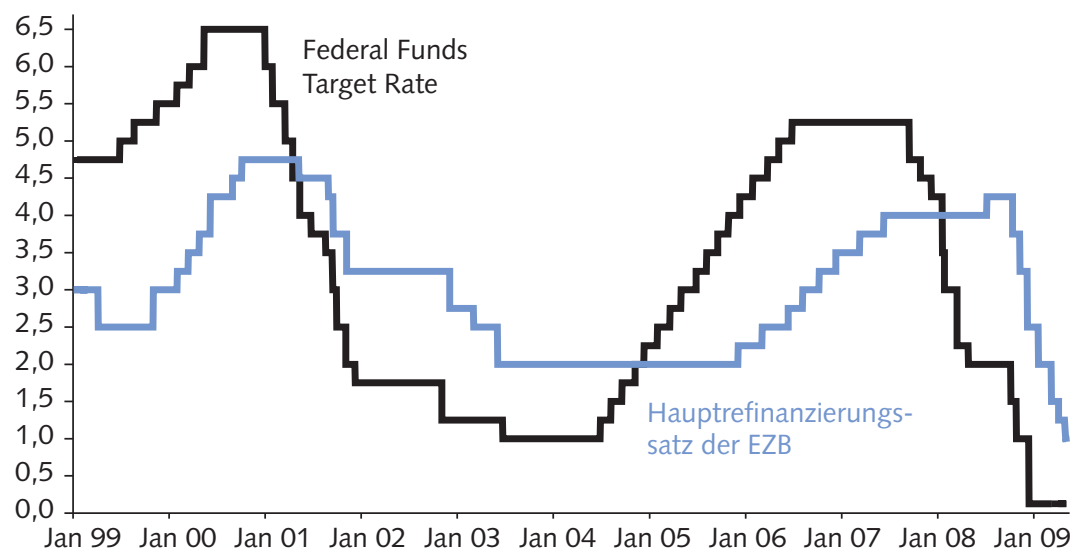

1 Seit dem 16.12.2008 beträgt die Intended Federal Funds Rate 0-0,25 \%,

Quelle: Europäische Zentralbank; Federal Reserve Board.

WSI MITTEILUNGEN

auf die mittelfristige Inflationsentwicklung keinen Einfluss gehabt hätte.

Zögerlich und schwach reagierte die EZB dann auf die sich in der ersten Jahreshälfte 2001 abzeichnende Eintrübung der binnenwirtschaftlichen Entwicklung und des weltwirtschaftlichen Umfeldes. Während die US-amerikanische Zentralbank als Reaktion auf die als zu stark angesehene Abflachung des Wachstums schon im Januar 2001 ihre Politik lockerte und die Leitzinsen bis zum Sommer bereits um 3 Prozentpunkte gesenkt hatte, reagierte die EZB auf die von ihr nach unten revidierten Wachstumserwartungen bis Ende August 2001 mit einer Zinssenkung von nur einem viertel Prozentpunkt. Bis Ende 2001 senkte die Fed die Zinsen weiter auf 1,75 \%, während die EZB die Leitzinsen nur auf 3,25\% zurücknahm, obwohl sich für 2001 und 2002 geringe Wachstumsraten abzeichneten (Europäische Zentralbank 2001, S. 59), die auch aus Sicht der EZB das Entstehen einer nennenswerten Produktionslücke im Euroraum implizierten.

Das lange Stillhalten der EZB trotz der deutlichen Eintrübung der Aussichten im Laufe des Jahres 2001 lag darin begründet, dass die EZB befürchtete, mit Zinssenkungen eine prozyklische Politik in Gang zu setzen, die sich angesichts der zurückliegenden Preisschübe bei Rohöl und Nahrungsmitteln in höheren Lohnforderungen niederschlagen könnte. So prognostizierten die nationalen und internationalen Organisationen zwar einen Abschwung für 2001, für 2002 aber bereits wieder einen
Aufschwung. Dies allein rechtfertigte jedoch keine geldpolitische Zurückhaltung: Da geldpolitische Maßnahmen relativ schnell wirken, ${ }^{7}$ hätte ein Aufschwung 2002 lediglich bewirkt, dass sich die Produktionslücke schneller geschlossen hätte. Das Risiko einer Übersteuerung war gering, zumal, wie auch die EZB damals betonte, die Lohnentwicklung moderat war.

Tatsächlich fiel der BIP-Anstieg im Jahre 2002 mit $0,9 \%$ noch geringer aus als erwartet und es kam erst 2005 wieder zu einem deutlichen Aufschwung. Die Arbeitslosenquote war auf knapp $9 \%$ gestiegen. Die EZB senkte zwar den geldpolitischen Leitzins zwischen Dezember 2001 und April 2003 um weitere 1,25 Prozentpunkte auf $2 \%$, wo sie ihn dann bis Ende 2005 belies, aber der Euro wertete in dieser Zeit so stark auf, dass sich die monetären Rahmenbedingungen insgesamt bis 2005 verschlechterten. So nahm der Außenwert des Euro zwischen Dezember 2001 und Dezember 2004 real effektiv um $25 \%$ und gegenüber dem US-Dollar um $50 \%$ zu.

Makroökonometrischen Schätzungen zufolge bewirken die Zinssenkungen um 1,25 Prozentpunkte einen Anstieg des Bruttoinlandsprodukts um etwa $0,6 \%$ in den ersten beiden Jahren, während die

\footnotetext{
Untersuchungen anhand mehrerer ökonometrischer Modelle zeigen, dass die Geldpolitik im Euroraum relativ zügig wirkt. Eine Zinssenkung hat demnach bereits nach einem halben Jahr einen signifikant positiven Einfluss auf das Bruttoinlandsprodukt (Europäische Zentralbank 2002a, S. 47ff.)
} 
Aufwertung des Euro das BIP um $2 \%$ senkte. ${ }^{8}$

\subsection{VERHALTENE GELDPOLITISCHE REAKTION AUF DIE WELTWIRT- SCHAFTSKRISE 2008/2009}

Ähnlich wie das Jahr 2000 war auch das Jahr 2007 geprägt von einer kräftigen $\mathrm{Zu}$ nahme des Bruttoinlandsproduktes, einem deutlichen Rückgang der Arbeitslosigkeit und einem starken Anstieg der Rohstoffpreise. Im folgenden Jahr hatte sich das Wachstum bereits auf 0,8\% abgeschwächt und 2009 folgte ein deutlicher Rückgang der gesamtwirtschaftlichen Produktion. War es 2000/2001 die Kombination eines starken Ölpreisanstiegs, des Platzens der Internetblase und der weltweit restriktiven Geldpolitik, die den Aufschwung jäh beendeten, so war es 2007/2008 die Kombination aus kräftigem Ölpreisanstieg, dem drastischen Einbruch der Immobilienpreise und der damit ausgelösten Finanzkrise bei ebenfalls weltweit angezogenen geldpolitischen Zügeln.

Das erste Anzeichen für die Finanzmarktkrise war der starke Anstieg der Zinsen am Interbankenmarkt (Geldmarkt) im August 2007. Die EZB reagierte auf den darin zum Ausdruck kommenden Vertrauensverlust zwischen den Banken zwar zügig, indem sie Refinanzierungsmittel zunehmend längerfristig zur Verfügung stellte. Die Fed senkte darüber hinaus aber auch die Zinsen. Knapp ein Jahr später, als sich die USA bereits im vierten Quartal der Rezession befanden und die Fed die Zinsen bereits um 2,75 Prozentpunkte gesenkt hatte, erhöhte die EZB den geldpolitischen Leitzins um einen viertel Prozentpunkt auf 4,25\%. Zu diesem Zeitpunkt befand sich der Euroraum im zweiten Quartal der Rezession. Noch stärker als der Leitzins - und zwar um 0,7 Prozentpunkte - hatten sich im ersten Jahr der Finanzkrise die durchschnittlichen Refinanzierungskosten der Banken bei der EZB erhöht, da die EZB zwar mehr Liquidität zur Verfügung stellte, aber deutlich weniger als von den Banken nachgefragt wurde (Arbeitskreis Konjunktur 2008, S. 25ff.).

Erst nach der nochmaligen Verschärfung der Finanzkrise durch die Insolvenz von Lehman Brothers im September 2008 ging die EZB zu einen expansiven Kurs über. Im Oktober senkte sie den geldpolitischen Leitzins um einen halben Prozentpunkt und hob zugleich die mengenmäßi- ge Beschränkung bei der Liquiditätsversorgung auf. Zugleich erweiterte sie den Kreis derjenigen, die sich bei ihr Liquidität verschaffen können, wie auch den der als Sicherheiten zulässigen Wertpapiere. Die Banken konnten sich nun gegen die Übertragung von Sicherheiten unbegrenzt Liquidität für einen Zeitraum von einer Woche bis zu sechs Monaten bei der EZB beschaffen, und zwar zu einem Zinssatz von 3,75\%. Angesichts der Unsicherheit über ihren eigenen Liquiditätsbedarf und die beschränkte Funktionsfähigkeit des Geldmarktes machten die Banken von dieser Möglichkeit stark Gebrauch und parkten die überschüssigen Mittel in der mit 3,25\% verzinsten Einlagefazilität der EZB. Es folgten weitere Zinssenkungen im November $(3,25 \%)$ und Dezember (2,5\%).

Zur Jahreswende 2008/2009 befand sich die Wirtschaft im Euroraum und weltweit im freien Fall. Die Produktion war rückläufig, die Aktienkurse brachen erneut ein und die Gefahr weiterer Bankenzusammenbrüche sowie einer Deflation war hoch. Während die Fed und andere bedeutende Zentralbanken bereits Ende 2008 auf eine Nullzinspolitik übergegangen waren und darüber hinaus mit dem direkten Kauf von Unternehmens- und Staatsanleihen (quantitative Lockerung) expansiv wirkten, behielten im EZB-Rat die Hardliner die Oberhand, die sich gegen „zu niedrige“ Zinsen aussprachen und Maßnahmen der quantitativen Lockerung eine Absage erteilten. Der Widerstreit im EZB-Rat führte im April 2009 zu der kuriosen Entscheidung, den Leitzins nicht wie von den Marktteilnehmern erwartet um 0,5 Prozentpunkte zu senken, sondern nur um 0,25 Prozentpunkte. Angesichts der von den Hardlinern gezogenen Untergrenze von $1 \%$ wurde so für die folgende Sitzung im Mai, bei der auch unkonventionelle Maßnahmen angekündigt werden sollten, ein Tippelschritt nach unten aufgehoben. ${ }^{9}$

Im Mai 2009 kam es dann vor dem Hintergrund erneut deutlich eingetrübter wirtschaftlicher Perspektiven - die OECD (2009) und die Gemeinschaftsdiagnose (2009) prognostizierten beispielsweise ein Schrumpfen des BIP um 4,1 \% bzw. 4,5 \% - doch zu weiterreichenden Maßnahmen. Trichet betonte explizit, dass der Leitzins in Zukunft auch unter den nunmehr geltenden Leitzins von $1 \%$ fallen könnte. Wie erwartet, wurde die Länge der Refinanzierungsgeschäfte auf zwölf Monate ausgeweitet. Mit der ebenfalls beschlossenen $\mathrm{Zu}-$ lassung der Europäischen Investitionsbank (EIB) zur Refinanzierung bei der EZB wurde dieser eine zinsgünstige Liquiditätsbeschaffung ermöglicht, was sich wiederum günstig auf ihre Kreditvergabekonditionen auswirken dürfte. Als quantitative Lockerung kann man den direkten Kauf von Pfandbriefen (Volumen $60 \mathrm{Mrd}$. €) ab Juli 2009 interpretieren. Zwar geht es der EZB primär um eine Entspannung in diesem Segment der von Banken emittierten und mit Hypotheken besicherten Wertpapiere, durch die keine zusätzliche Liquidität in Umlauf gebracht werden soll. Allerdings ist zu erwarten, dass entsprechende sterilisierende Offenmarktverkäufe von Wertpapieren erst nach einer Normalisierung der Situation zwischen den Banken erfolgen werden, da sich die Banken aktuell beliebig viel Liquidität bei der EZB zu dem niedrigen Zinssatz von $1 \%$ besorgen können.

Ähnlich wie in der Schwächephase ab 2001 hat die EZB auch auf die im Jahr 2008 beginnende Krise zögerlich reagiert und hat auch Mitte 2009 ihren auf Null begrenzten zinspolitischen Spielraum nicht

8 Nach Berechnungen der Gemeinschaftsdiagnose mit dem Weltmodell des Forschungsinstituts Oxford Economic Forecasting entspricht die realwirtschaftliche Wirkung einer $10 \%$ igen Abwertung des US-Dollar gegenüber dem Euro der einer Zinssenkung im Euroraum um einen Prozentpunkt (Gemeinschaftsdiagnose 2003, S. 229).

9 Vertreter der EZB reagierten auf Kritik an ihrer zögerlichen Haltung gelegentlich mit dem Hinweis, dass die EZB trotz höherer Leitzinsen ebenso wirksam expansiv agiere wie die Fed und verwiesen dabei auf die Geldmarktzinsen am Interbankenmarkt sowie auf die deutliche Verlängerung der Bilanz des Europäischen Systems der Zentralbanken (ESZB). Die Dreimonatszinsen im Euroraum lagen aber ab Anfang 2008 deutlich über den US-amerikanischen. Die US-amerikanischen Zwölf- und die Sechsmonatszinsen lagen ebenfalls zwischen November 2007 bzw. Januar 2008 und Februar 2009 deutlich, teilweise um mehr als 2 Prozentpunkte, unter denen des Euroraums. Erst seitdem liegen die des Euroraums teilweise geringfügig niedriger, was als Erfolg der längerfristigen Liquiditätsbereitstellung zu werten ist. Auch waren die Bilanzveränderungen der beiden Zentralbanken von der Größe und den Bestimmungsgründen her sehr unterschiedlich: Die Bilanzsumme des ESZB war Mitte April 2009 um knapp 60 \% höher als zwei Jahre zuvor, die der Fed um gut $150 \%$. Beim ESZB ist lediglich ein Drittel der Verlängerung auf die Geldpolitik zurückzuführen; demgegenüber geht der Löwenanteil der Bilanzverlängerung der Fed auf eine aktive Politik der Fed zurück: Allein das Programm zum Kauf von Commercial Papers bewirkte eine Bilanzverlängerung um $28 \%$, die geldpolitischen Operationen im engeren Sinne einer Verlängerung um $50 \%$ und der direkte Kauf von Wertpapieren einen Anstieg um $18 \%$. 
ausgeschöpft, obwohl die Zinsen sogar negativ sein müssten, um der aktuellen Situation gerecht zu werden. ${ }^{10}$

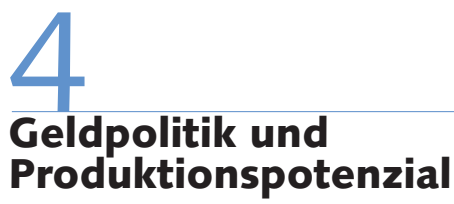

Das Problem einer Geldpolitik, die tendenziell bestrebt ist „to err on the side of caution" (Lamfalussy 1997), ist in der gegenwärtigen Situation einer Finanz- und Wirtschaftskrise, dass sich dadurch das Risiko einer Deflation erhöht, deren Überwindung ungleich schwerer ist als die Bändigung aufkommender Inflationstendenzen. Aber auch in „normalen“ Zeiten ohne Deflations- und Stagnationsgefahr kann sich eine Geldpolitik mit restriktivem Bias negativ auswirken. So kann beispielsweise die Annahme eines geringen Potenzialwachstums zu einer sich selbst erfüllenden Prophezeiung werden. Dies dürfte in der ersten Hälfte des Jahrzehnts passiert sein, wobei die Finanzpolitik, insbesondere in Deutschland, eine Mitverantwortung trägt. Das Potenzialwachstum einer Volkswirtschaft kann nicht mit einem hohen Grad an $\mathrm{Zu}$ verlässigkeit geschätzt werden, auch wenn komplizierte Verfahren angewendet werden. In der Regel werden daher lediglich statistische Verfahren angewandt, die den Trend der Vergangenheit fortschreiben. Die tatsächliche Entwicklung am aktuellen Rand spielt in diesen Verfahren eine dominante Rolle, wodurch es zu häufigen Revisionen kommt. ${ }^{11}$ Entsprechend bewirkte die länger währende Schwächephase bis 2005 eine Revision des Produktionspotenzials bzw. des Potenzialwachstums des Euroraums nach unten. Beispielhaft lässt sich dies an den Revisionen des IMF zeigen: Im Herbst 2001 bezifferte der IMF im World Economic Outlook die Produktionslücke, d.h. die Abweichung des Bruttoinlandsproduktes vom Produktionspotenzial in Prozent des Produktionspotenzials, des Jahres 2000 mit $-0,1 \%$. Mit anderen Worten, Bruttoinlandsprodukt und Produktionspotenzial entsprachen sich in etwa. Im April 2009 wies der IMF demgegenüber für das Jahr 2000 eine positive Produktionslücke in Höhe von $2 \%$ des BIP aus. Zu einem geringen Teil ist diese Revision auf Revisionen des BIP zurückzuführen. So stieg das BIP im Jahre 2000 um 3,9 \% und nicht, wie 2001 veranschlagt, um 3,5\%.
Ganz wesentlich aber beruht die Revision auf dem geringen Wachstum bis 2005 und der aktuellen Krise, die das Potenzial aus statistischen Gründen nach unten ziehen. Die damalige Lohnentwicklung passt eher in das Bild einer annähernd geschlossenen Produktionslücke, nicht in das einer Überhitzung. Die Löhne und Gehälter je Beschäftigten nahmen im Jahr 2000 um 2,5\% zu (EZB 2002b, S. ${ }^{*} 48$ ). Bei einem durchschnittlichen mittelfristigen Anstieg der Arbeitsproduktivität von über $1 \% \mathrm{im} \mathrm{Eu-}$ roraum und einer Zielinflationsrate der EZB in Höhe von 1,9 \% ging von den Löhnen somit ein leicht dämpfender Effekt aus, was nur schwerlich als Zeichen für eine konjunkturelle Überhitzung gedeutet werden kann.

Wegen der begrenzten Möglichkeiten, das Produktionspotenzial und das Potenzialwachstum hinreichend genau zu schätzen, ist es sinnvoll, wenn die Geldpolitik in Zeiten ruhiger Preisperspektiven tendenziell expansiv wirkt, um die Grenzen des Wachstums zu testen. Dabei geht es nicht darum, eine inflationäre Politik zu betreiben. Spätestens seit den 1990er Jahren besteht in der Wissenschaft und auch in weiten Teilen der Politik Konsens darüber, dass Preisniveaustabilität eine wichtige Voraussetzung für eine stabile wirtschaftliche Entwicklung bildet. Aus diesem Grund sind zahlreiche Zentralbanken explizit oder auch implizit zu einer Vorgabe von Inflationszielen und zu einer Strategie des Inflation Targeting übergegangen. Bei Wahrung der Preisstabilität sollte die Zentralbank aber expansiv genug wirken, um einen Rückgang der hohen Arbeitslosigkeit zu ermöglichen, insbesondere indem sie bei höheren Wachstumsraten und geringer Inflation geldpolitisch abwartet. Sofern das Ziel der Preisstabilität nicht gefährdet ist, ist eine solche Orientierung durchaus auch im EU-Vertrag angelegt. ${ }^{12}$ Eine solche Ausrichtung der Geldpolitik trägt dem Umstand Rechnung, dass das Potenzialwachstum bzw. die inflationsstabile Arbeitslosenquote (NAIRU) keine exogen gegebenen Größen sind, sondern von der tatsächlichen, auch von der Geldpolitik beeinflussten BIP- und Beschäftigungsentwicklung bestimmt werden. ${ }^{13}$ Die Integration eines nennenswerten Teils der Arbeitslosen in den Arbeitsprozess im Zuge eines Aufschwungs und die Anpassung des Kapitalstocks an das erhöhte effektive Arbeitsangebot könnten das Produktionspotenzial steigern und über mehrere Jahre ein über- durchschnittliches Wachstum ermöglichen. Zudem weist die OECD (2006, S. 49) darauf hin, dass ein lang anhaltender Aufschwung tendenziell mit einer steigenden Erwerbsbeteiligungsquote einhergeht, die ihrerseits wiederum das Arbeitskräfteangebot und das Produktionspotenzial erhöht.

Die Wirtschaftsforschungsinstitute haben für die Jahre 2008 bis 2013 für den Euroraum ein durchschnittliches Wachstum von $0 \%$ vorhergesagt (Gemeinschaftsdiagnose 2009, S. 70) und zugleich das Potenzialwachstum deutlich heruntergesetzt. Noch im Frühjahr 2008 war das Potenzialwachstum der EU mit 2,3 \% beziffert worden, ${ }^{14}$ das Deutschlands mit 1,6 \%; nunmehr werden $1,5 \%$ bzw. $0,9 \%$ veranschlagt. Um eine derart desolate Entwicklung und den korrespondierenden Anstieg der Arbeitslosigkeit zu verhindern, sollte die EZB hinreichend lang expansiv wirken. Während eine Ausstiegsstrategie aus der derzeit stark expansiven Geld- und Fiskalpolitik unbedingt erforderlich ist, muss sich der Zeitpunkt ihrer Umsetzung an der wirtschaftlichen Entwicklung orientieren. Wird der expansive Kurs zu früh verlassen, droht nicht nur Stagnation, sondern eine erneute Rezession. Neben einer zu frühzeitig implementierten Ausstiegsstrategie stellt auch die Entwicklung des Ölpreises einen möglichen wirtschaftspolitischen

10 So legt die von den führenden Wirtschaftsforschungsinstituten verwendete Taylor-Regel gar einen Zins von aktuell $-2 \%$ sowie ein Absinken auf $-4,5 \%$ bis Mitte 2010 nahe (Gemeinschaftsdiagnose 2009, S. 88). Der Financial Times zufolge kommt eine interne Studie der Fed zu dem Schluss, dass der angemessene Zins in den USA bei $-5 \%$ liegt (Guhu 2009)

11 Siehe auch Orphanides/Williams (2002), die diese Diskrepanz zwischen Potenzialschätzungen in Echtzeit und im Rückblick für die Vereinigten Staaten untersuchen, sowie die Studie von Döpke (2004) für Deutschland.

12 So steht im EU-Vertrag: "Soweit dies ohne Beeinträchtigung des Zieles der Preisstabilität möglich ist, unterstützt die EZB die allgemeine Wirtschaftspolitik in der Gemeinschaft, um zur Verwirklichung der in Artikel 2 festgelegten Ziele der Gemeinschaft [...eine harmonische, ausgewogene und nachhaltige Entwicklung des Wirtschaftslebens, ein hohes Beschäftigungsniveau...] beizutragen. " Art. 105 (1) Konsolidierte Fassung des Vertrags zur Gründung der Europäischen Gemeinschaft.

13 Die übliche Annahme einer langfristigen Neutralität der Geldpolitik, der hiermit widersprochen wird, ist weder theoretisch noch empirisch zwangsläufig, vgl. beispielsweise: Ball/Mankiw (2002); Solow (2000); DeGrauwe/Costa Storti (2007).

14 Für den Euroraum weist die Gemeinschaftsdiagnose die Potenzialschätzung nicht aus, erwähnt aber, dass diese niedriger liegt als jene für die EU. 
Stolperstein dar. Mit der Erholung der globalen Wirtschaft wird der Ölpreis voraussichtlich anziehen. Der damit einhergehende Anstieg der Inflationsrate sollte von der Zentralbank den gängigen geldpolitischen Überlegungen entsprechend hingenommen werden, sofern er keine Zweitrundeneffekte (Preis-Lohn-Spirale) auslöst (Tober/Zimmermann 2009).

\section{5}

Einfach war die geldpolitische Aufgabe in den ersten zehn Jahren des Euroraums nicht: Die EZB war mit Ölpreisschocks konfrontiert, mit synchronen weltwirtschaftlichen Abschwüngen, starken Schwankungen des Außenwerts des Euro und einer heterogenen Entwicklung innerhalb des Euroraums (siehe auch Beitrag von Ulrich Fritsche in diesem Heft). Insgesamt war die Geldpolitik der EZB insofern erfolgreich, als sie für Preisstabilität gesorgt hat und an den Märkten ein hohes Vertrauen genießt, was in den geringen Inflationserwartungen und Kapitalmarktzinsen zum Ausdruck kommt. Grundsätzlich ist ihre Strategie des Inflation Targeting zudem geeignet, ein hohes Maß an Stabilität zu gewährleisten. Allerdings offenbarte die EZB in ihren Verlautbarungen und in ihrem Handeln einen restriktiven Bias, der mit dazu beitrug, dass das Wirtschaftspotenzial im Euroraum in den vergangenen zehn Jahren zumeist nicht ausgeschöpft wurde. Der restriktive Bias kam dabei in der Definition von Preisstabilität als Obergrenze zum Ausdruck wie auch in der Annahme eines relativ geringen Potenzialwachstums und der zögerlichen Reaktion auf die beiden Wirtschaftseinbrüche in dieser Zeit.

In der Vergangenheit haben regionale Fehlentwicklungen die Geldpolitik im Euroraum erschwert. Zum Teil war die Lohnentwicklung im Euroraum nur deshalb stabilitätsgerecht, weil die gedämpfte Lohnentwicklung in Deutschland stabilitätswidrige Entwicklungen beispielsweise in Spanien kompensierte. Auf Fehlentwicklungen in einzelnen Ländern sollte die EZB gezielt hinweisen und den Makroökonomi- schen Dialog zum Zwecke einer Kurskorrektur nutzen (siehe auch den Beitrag von Hallwirth/Koll in diesem Heft), zumal diese Fehlentwicklungen in der Zukunft Anpassungsprozesse nach sich ziehen können, die dann erneut die gesamte Währungsunion belasten. Für eine stabile, inflationsfreie wirtschaftliche Entwicklung bei möglichst geringer Arbeitslosigkeit ist eine vernünftige Aufgabenverteilung der verschiedenen Makropolitikbereiche erforderlich. Regionalen Fehlentwicklungen - ob Schwäche oder Überhitzung - kann in der Währungsunion vor allem fiskalpolitisch begegnet werden. Je geringer der Lohndruck, desto expansiver kann und muss die Geldpolitik sein. Gelingt es in den kommenden Jahren im gesamten Euroraum die Voraussetzungen für eine stabilitätsgerechte Lohnentwicklung zu schaffen, so könnte sich mithilfe einer expansiven Wirtschaftspolitik eine deutlich kräftigere wirtschaftliche Entwicklung entfalten als derzeit vielfach erwartet wird. Diese Entwicklung wäre zudem balancierter als in den vergangenen zehn Jahren.

\section{LITERATUR}

Arbeitskreis Konjunktur (2008): Am Rande des Abgrunds. Prognose der wirtschaftlichen Lage 2009, IMK Report 35

Artis, M./Mizen, P./ Kontolemis, Z. (1998): Inflation Targeting: What Can the ECB Learn From the Recent Experience of the Bank of England? in: The Economic Journal 108, S. 1810-25

Ball, L./Mankiw, N. G. (2002): The NAIRU in Theory and Practice, in: Journal of Economic Perspectives 4, S. 115-136

Bernanke, B. (2004): The logic of monetary policy, Remarks before the National Economists Club, December 2ed, Washington, D. C.

Bernanke, B. S./Mihov, I. (1997): What does the Bundesbank target? in: European Economic Review 6, S. 1025-1053

Bernanke, B. S./Mishkin, F. S. (1997): Inflation Targeting: A New Framework for Monetary Policy?, NBER Working Paper 5893, S. 1

Brand, C./Cassola, N. (2000): A Money Demand System for Euro Area M3, ECB Working Paper 39, Frankfurt/M.

Coenen, G./Vega, J.-L. (1999): The Demand for M3 in the Euro Area, ECB Working Paper 6, Frankfurt/M.

DeGrauwe, P./Storti, C. (2007): Monetäre Politik und reale Ökonomie. Aufschwung für Deutschland, in: Schettkat, R./Langkau, J. (Hrsg.): Plädoyer international renommierter Ökonomen für eine bessere Wirtschaftspolitik, Bonn, S. 49-80
Döpke, J. (2004): Real-time data and business cycle analysis in Germany. Discussion Paper, Studies of the Economic Research Centre 11 Duisenberg, W. F. (1999): The Eurosystem's Monetary Policy Strategy: The first Year's Experience. Rede auf dem Joint Congress of the Federations, EUROFINAS and LEASEUROPE, Paris, 11. Oktober

Europäische Zentralbank (1999): Monatsbericht Dezember, Frankfurt/M. Europäische Zentralbank (2000): Monatsbericht Dezember, Frankfurt/M. Europäische Zentralbank (2001): Monatsbericht Dezember, Frankfurt/M. Europäische Zentralbank (2002a): Monatsbericht Oktober, Frankfurt/M. Europäische Zentralbank (2002b): Monatsbericht Dezember, Frankfurt/M.

Europäische Zentralbank (2003): Die geldpolitische Strategie der EZB, Pressemitteilung vom 8. Mai, Frankfurt/M.

Europäische Zentralbank (2006): Monatsbericht September, Frankfurt/M. Gemeinschaftsdiagnose (1999): Die Lage der Weltwirtschaft und der deutschen Wirtschaft im Herbst 1999, in: IWH, Wirtschaft im Wandel 14, Halle

Gemeinschaftsdiagnose (2000): Die Lage der Weltwirtschaft und der deutschen Wirtschaft im Herbst 2000, in: DIW-Wochenbericht 43 Gemeinschaftsdiagnose (2003): Die Lage der Weltwirtschaft und der deutschen Wirtschaft im Frühjahr 2003, in: DIW-Wochenbericht 16, Berlin 
Gemeinschaftsdiagnose (2008): Deutschland am Rande einer Rezession - Gemeinschaftsdiagnose Herbst, IMK Report 32, Düsseldorf Gemeinschaftsdiagnose (2009): Im Sog der Weltwirtschaft - Gemeinschaftsdiagnose Frühjahr, IMK Report 37, Düsseldorf

Guhu, K. (2009): Fed study puts ideal interest rate at $-5 \%$, in: Financial Times, London, 27. April

International Monetary Fund (2000a): Staff Report on the Monetary and Exchange Rate Policies of the Euro Area; Supplement Updating Information on Recent Economic Developments and Policies in the Euro Area; Supplement on EU Trade Policies; Public Information Notice Following Consultation; Two Statements by Representatives on the IMF Executive Board for France, on Behalf of Euro-Area and EU Members, IMF Staff Country Report 00/146, November International Monetary Fund (2000b): World Economic Outlook, Washington

Kakes, J. (2000): Monetary Transmission and Business Cycle Asymmetry. Kredit und Kapital 2, S. 182-197

Kuzin, V./Tober, S. (2004): Asymmetric Effects of Monetary Policy in Germany, DIW-Diskussionspapier 351, Berlin

Lamfalussy, A. (1997): The operation of monetary policy in stage three of EMU. Address by Alexandre Lamfalussy, President of the European Monetary Institute, to the Euromoney Conference in New York, 30. April Neumann, M. J. M. (1998): On the Choice of a Strategy for the European Central bank's Monetary Policy, Position Paper for EMU-Monitor, June Noyer, C. (2000): Monetary Policy-Making in the Euro Area. Rede auf dem Pareto Fonds' 2000 Economic Forum, Oslo, 23. März

OECD (2000): Economic Outlook, Paris

OECD (2006): Employment Outlook - Boosting Jobs and Income, Paris OECD (2009): Interim Economic Outlook, März, Paris
Orphanides, A./Williams J. C. (2002): Robust Monetary Policy Rules with Unknown Natural Rates, in: Brookings Papers on Economic Activity 2, S. 63-126

Sachverständigenrat zur Begutachtung der gesamtwirtschaftlichen Entwicklung (SVR) (1998): Weitreichende Entscheidungen, Jahresgutachten 1998/99, Stuttgart

Schumacher, C./Tober, S. (1999a): Unzureichende Indikatoreigenschaften der Geldmenge: Konsequenzen für die geldpolitische Strategie der EZB, IWH, Forschungsberichte 9

Schumacher, C./Tober, S. (1999b): Europäische Geldpolitik - Einschwenken auf neutralen Kurs, IWH, in: Wirtschaft im Wandel 15, S. 12-18

Solow, R. M. (2000): Unemployment in the United States and in Europe: A contrast and the reasons, Working Paper 231, CESifo

Solow, R. M. (2007): Die Beschränktheit der makroökonomischen Diskussion überwinden, in: Schettkat, R./Langkau, J. (Hrsg.): Aufschwung für Deutschland. Plädoyer international renommierter Ökonomen für eine bessere Wirtschaftspolitik, Bonn, S. 35-47

Svensson, L. (1997): Inflation Forecast Targeting: Implementing and Monitoring Inflation Targets, in: European Economic Review 41, S. 10251053

Svensson, L. (2008): What have economists learned about monetary policy over the past 50 years?, Speech at the conference: Monetary policy over fifty years, Deutsche Bundesbank, 21. September, Frankfurt/M.

Svensson, L./Gerlach, S. (2002): Money and Inflation in the Euro Area: A Case for Monetary Indicators?, CEPR Discussion Paper 3392, London Tober, S. (2000): Geldpolitik in besonderer Verantwortung für den Aufschwung, DIW-Wochenbericht 47, S. 801-806

Tober, S./Zimmermann, T. (2009): Monetary Policy and Commodity price shocks, in: Intereconomics July/August, S. 231-237 